\title{
Floquet Solitons and Dynamics of Periodically Driven Matter Waves with Negative Effective Mass
}

\author{
Matthew Mitchellø, ${ }^{1}$ Andrea Di Carli®, ${ }_{1}^{1}$ Germán Sinuco-León $\odot,{ }^{2}$ Arthur La Rooij®, ${ }^{1}$ \\ Stefan Kuhr $\odot,{ }^{1}$ and Elmar Haller ${ }^{1}$ \\ ${ }^{1}$ Department of Physics and SUPA, University of Strathclyde, Glasgow G4 ONG, United Kingdom \\ ${ }^{2}$ Department of Chemistry, Durham University, Durham DH1 3LE, United Kingdom
}

(Received 1 June 2021; revised 2 September 2021; accepted 26 October 2021; published 10 December 2021)

\begin{abstract}
We experimentally study the dynamics of weakly interacting Bose-Einstein condensates of cesium atoms in a $1 \mathrm{D}$ optical lattice with a periodic driving force. After a sudden start of the driving, we observe the formation of stable wave packets at the center of the first Brillouin zone (BZ) in momentum space, and we interpret these as Floquet solitons in periodically driven systems. The wave packets become unstable when we add a trapping potential along the lattice direction, leading to a redistribution of atoms within the BZ. The concept of a negative effective mass and the resulting changes to the interaction strength and effective trapping potential are used to explain the stability and the time evolution of the wave packets. We expect that similar states of matter waves exist for discrete breathers and other types of lattice solitons in periodically driven systems.
\end{abstract}

DOI: $10.1103 /$ PhysRevLett.127.243603

Ultracold quantum gases in optical lattices have proven to be excellent tools for the experimental study of novel quantum systems $[1,2]$. In particular, optical lattices with periodic driving forces provide detailed control of tunneling between lattice sites and band structures with new, intriguing features [3]. Examples are the demonstration of a dynamically driven quantum phase transition between a bosonic Mott insulator and a superfluid [4], kinetic frustration on a triangular lattice [5], artificial magnetic fields [6-9], and topological band structures [10-12]. The systems are commonly described by the Floquet formalism, which maps the periodic driving to a time-independent Hamiltonian [3,13].

Essential for many experiments is the control of the dispersion relation between quasimomentum and energy in the energetically lowest lattice band $[14,15]$. In particular, increasing the amplitude of the driving force can invert the dispersion curve, turning the energetically highest states at the edge of the first Brillouin zone (BZ) into the energetically lowest states [16-18]. The resulting evolution of atoms from the center of the BZ toward its edge [19] is surprising because the two states in the BZ are not connected by a continuous change of driving strength. Other mechanisms such as interactions, external forces, or cooling mechanisms are required as an explanation. Understanding this time evolution is instrumental, e.g., for quantum simulation using ultracold atoms in driven systems and the creation of Floquet condensates [19-21].

In this Letter, we experimentally study the evolution of a weakly interacting Bose-Einstein condensate (BEC) of cesium atoms in a periodically driven 1D lattice potential after a sudden start of the driving. We demonstrate that matter waves in periodically driven systems can be stable at the center of the BZ despite an inverted dispersion curve, forming time-averaged gap solitons $[22,23]$ with a negative effective mass. Those Floquet solitons have recently been demonstrated in optics using photonic waveguide arrays [24], and they have been predicted to exist also for matter waves [24-28]. We demonstrate the formation and stability of those states in momentum and position space over a duration of $1 \mathrm{~s}$.

We first provide a systematic study of the dynamics of matter waves for various driving strengths, demonstrating the stability of ground states and the decay of excited states in the BZ. We observe the typical instability of atoms at the center of the BZ for an inverted dispersion curve; however, in our weakly interacting system, this evolution toward the edge of the BZ is caused by an external trapping potential. We provide an intuitive explanation for this effect by extending the concepts of group velocity and effective mass to periodically driven systems [29]. Atoms in states with a negative effective mass are unstable due to an effectively expulsive trapping potential along the lattice direction [30] that pushes them toward the edge of the BZ. Removing this trapping potential allows us to create and study Floquet solitons.

Our experimental starting point is a magnetically levitated BEC in a crossed-beam optical dipole trap and a vertical optical lattice potential at a wavelength $\lambda=1064 \mathrm{~nm}$ and lattice spacing $d_{L}=\lambda / 2$ [Fig. 1(a)]. Depending on the required driving frequency, we apply the driving force $F(t)$ by either modulating a vertical magnetic 

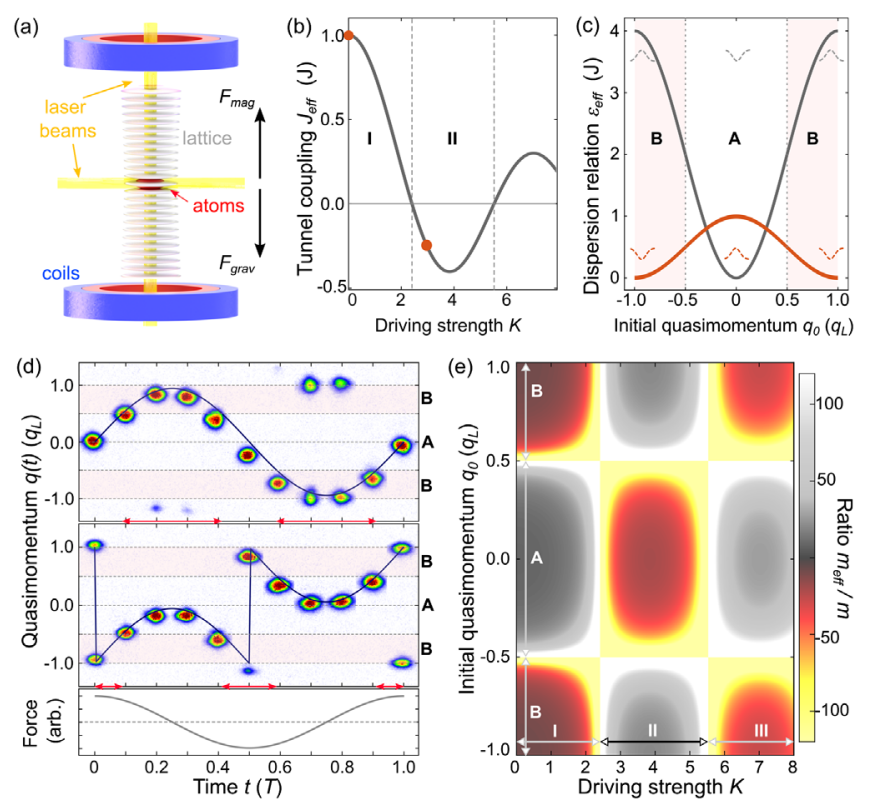

FIG. 1. Experimental setup, micromotion, and effective mass. (a) Experimental setup. (b) Effective tunnel coupling $J_{\text {eff }}$ as a function of driving strength $K$ with circles at $K=0$ and $K=2.97$. (c) Dispersion relation for $K=0$ (gray) and $K=$ 2.97 (red). Dashed lines indicate the effective trapping potential in regions A and B. (d) Absorption images showing the micromotion of atoms for $q_{0}=0$ (top) and $q_{0}=q_{L}$ (middle) with driving force $K=2.97$ and driving period $T$. Red arrows indicate time intervals with the wave packet in region B. (e) Ratio of effective mass $m_{\text {eff }}\left(K, q_{0}\right)$ to real mass $m\left(V=12 E_{r}\right)$.

field gradient or by periodically shaking the position of the lattice sites with detuned laser beam frequencies [19]. For fast driving, we avoid parametric and interband excitations [31-35] by using shaking frequencies with excitation energies in the first band gap of the lattice. Further details about our setup and the parameters of our measurements are presented in the Supplemental Material [36].

We use a semiclassical description of a wave packet in the lowest lattice band to interpret our results. A driven wave packet that is initially localized at a Bloch state with quasimomentum $q_{0}$ moves through the $\mathrm{BZ}$ according to the acceleration theorem $q(t)=q_{0}+\int_{0}^{t} F\left(t^{\prime}\right) d t^{\prime}$ [29], with $F(t)=F_{0} \cos (\omega t)$, where $F_{0}$ is the amplitude of the force and $\omega$ is the driving frequency. In the lowest band, this socalled micromotion is well defined by the parameters $q_{0}$ and the dimensionless driving strength $K=F_{0} d_{L} /(\hbar \omega)$ [29]. We show examples of this motion in Fig. 1(d) for $q_{0}=0$ and $q_{0}=q_{L}=\hbar \pi / d_{L}$.

Time averaging the energy over one period of the micromotion provides an effective dispersion relation $[3,13]$

$$
\epsilon_{\mathrm{eff}}\left(K, q_{0}\right)=-2 J_{\mathrm{eff}}(K) \cos \left(\frac{\pi}{q_{L}} q_{0}\right)
$$

with an effective tunneling matrix element $J_{\text {eff }}(K)=$ $J \mathcal{J}_{0}(K)$, where $J$ is the tunneling matrix element and $\mathcal{J}_{0}(K)$ is the zeroth order Bessel function. We label the regions with positive and negative $J_{\text {eff }}$ with I and II $\left(0 \leq K_{I}<2.4 \leq K_{I I} \leq 5.5\right)$ in Fig. 1(b), and the regions with quasimomenta close to the center $\left(\left|q_{0}\right|<0.5 q_{L}\right)$ and close to the edge $\left(\left|q_{0}\right| \geq 0.5 q_{L}\right)$ of the BZ with A and B in Fig. 1(c). The inversion of $\epsilon_{\text {eff }}\left(K, q_{0}\right)$ appears when states with $q_{0}$ in region A spend a large fraction of their micromotion in region $\mathrm{B}$, gaining a larger average energy than states with $q_{0}$ in region $\mathrm{B}$ [e.g., top of Fig. 1(d)]. The argument of $\epsilon_{\text {eff }}$ is the initial quasimomentum $q_{0}$ at the start of a driving period $T=2 \pi / \omega$, and experimental measurements need to probe the system stroboscopically at integer multiples of $T$.

In analogy to nondriven lattice systems, we use the effective dispersion relation to define the effective inertial mass $m_{\text {eff }}$ of a wave packet

$$
\begin{aligned}
m_{\mathrm{eff}}\left(K, q_{0}\right) & =\left[\partial_{q_{0}}^{2} \epsilon_{\mathrm{eff}}\left(K, q_{0}\right)\right]^{-1} \\
& =\left[2 J_{\mathrm{eff}}(K)\left(\frac{d_{L}}{\hbar}\right)^{2} \cos \left(\frac{\pi}{q_{L}} q_{0}\right)\right]^{-1} .
\end{aligned}
$$

The effective mass determines the spreading of the wave packet $[42,43]$ and its response to an external force [30]. Periodic driving always increases $\left|m_{\mathrm{eff}}\right|$ and it inverts the signs of $m_{\mathrm{eff}}$ in region II [Fig. 1(e)].

We first demonstrate the stability of the ground states for $K$ in regions I and II. The momentum distribution of the ground state in region I is centered at $q_{0}=0$, and we use a stationary wave packet to study the ground state for $K=1.96$. In region II, the ground state is centered at $q_{0}=q_{L}$, and we accelerate the wave packet with a magnetic field gradient to the edge of the BZ before driving with $K=2.97$ [36]. The driving force is applied by shaking the lattice $\left[V=12.0(5) E_{r}, \omega=2 \pi \times 1 \mathrm{kHz}\right]$, while keeping the vertical trapping frequency $\omega_{z}=$ $2 \pi \times 8.2(1) \mathrm{Hz}$ unchanged. We measure the real momentum distribution instead of the quasimomentum to avoid difficulties when band mapping in a shaking lattice (see Supplemental Material, Sec. A [36]). No significant change of the momentum distribution is observed over $600 \mathrm{~ms}$, except for a loss of $25 \%$ of the atoms, indicating low heating rates in the ground state [Figs. 2(a) and 2(b)].

The time evolution of the state with $q_{0}=0$ changes drastically when we increase $K$ to cross into region II. For $J_{\text {eff }} \approx 0$, the strong suppression of tunneling leads to dynamic localization in position space [16-18]. In addition, we observe the formation and spreading of a pattern of density peaks in the momentum distribution [Fig. 2(c)]. Without tunneling, the system consists of an array of independent BECs, each experiencing a different time evolution of its phase. The interference pattern that forms after releasing those BECs from the lattice creates the momentum distribution, which can show revivals and 

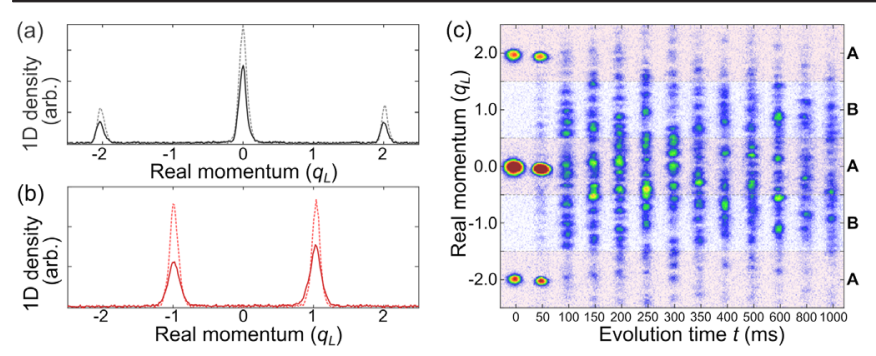

interactions ([36], Sec. B). For a quantitative analysis of the time evolution, we measure the ratio between the number of atoms in region $\mathrm{A}$ and the total atom number, $R(t)=N_{A}(t) / N_{\text {tot }}(t)$. The ratio $R(t)$ decreases from close to 1 (all atoms in region A) to values between 0.5 (uniform distribution) and 0.2 (localization in region B) [Fig. 2(f)].

The concept of an effective mass provides an intuitive explanation for the observed dynamics. Quenching the driving strength from $K=0$ to region II switches $m_{\mathrm{eff}}$ for the $q_{0}=0$ state from positive to negative, with important consequences for the time evolution. The evolution of a wave packet with $m_{\text {eff }}<0$ is identical to a wave packet with positive effective mass but with sign changes of the external potential and the interactions [30,47]. Driving with $K$ in region II effectively inverts the trapping potential for states with $q_{0}$ in region A. Atoms in those states are no longer trapped by the external potential but accelerated away from the trap center. Atoms in states in region B, however, are trapped due to their positive effective mass.

To simulate the complete dynamics, we numerically integrate the discrete nonlinear Schrödinger equation for renormalized lattice parameters

$$
i \hbar \partial_{t} \psi=(1-i \Lambda) H_{\mathrm{eff}} \psi,
$$

FIG. 2. Time evolution of the wave packet. (a) Momentum distributions of ground states for $t=0$ (dashed lines) and $t=$ $600 \mathrm{~ms}$ (solid lines) for $K=1.96, q_{0}=0$ and (b) for $K=2.97$, $q_{0}=q_{L}$. (c) Time evolution in momentum space with $m_{\text {eff }}<0$ for suppressed tunneling $J_{\text {eff }}=-0.01 J(K=2.43)$ and (d) for $J_{\text {eff }}=-0.34 J(K=3.26)$. Red patches indicated the regions A. (e) Numerical simulation with experimental parameters of (d). (f) Evolution of $N_{\text {tot }}$ and ratio $R$ for the measurement in (d). Solid lines are parabolic and exponential fits ([36], Sec. C). (g) Decay time $t_{1}$ for increasing trap periods in experiment (red circles) and simulation (blue squares).

temporal Talbot effects, depending on the time evolution of the phases [44]. Such interference patterns have been demonstrated for matter waves without driving [45,46], and we believe that the density peaks in our measurement are formed by a similar mechanism in a driven system. The patterns are fluctuating for experimental runs with identical control parameters, likely from increased technical noise due to the driving force; hence we show averaged images in Fig. 2 (see Supplemental Material, Sec. C [36] for images without averaging).

For stronger tunneling, $J_{\text {eff }}=-0.34 J(K=3.26)$, we observe that atoms move from region $\mathrm{A}$ to region $\mathrm{B}$ at the edge of the BZ [Fig. 2(d)], indicating a change of the micromotion from $q_{0}=0$ toward the state $q_{0}=q_{L}$ [Fig. 1(d)]. While it is experimentally straightforward to enforce this change with external forces, the mechanism is more intricate for periodic driving without net momentum transfer. We exclude instabilities, relaxation, and energy minimization in the band as explanations by demonstrating the stability of wave packets without driving for weak

with an effective Hamiltonian $H_{\text {eff }}$ in the tight-binding approximation ([36], Sec. D), a wave function $\psi$, and a phenomenological damping coefficient $\Lambda$ [48,49]. The simulation shows two stages in the time evolution [Fig. 2(e)], i.e., the initial spreading and fragmentation of the wave packet, and the subsequent slow localization of the wave packet in region B. For weak interactions, the initial spreading is dominated by the trapping potential, which accelerates the atoms and causes a rapid reduction of $R(t)$. The duration $t_{1}$ for $R(t)$ to drop to 0.7 increases with the trap period $2 \pi / \omega_{z}$ [Fig. $2(\mathrm{~g})$ ]. We find good agreement between simulation and experiment when we add small atom number fluctuations to the initial state to simulate finite temperature and residual nonadiabaticity during lattice loading ([36], Sec. D).

The second stage in the time evolution is controlled by the damping parameter $\Lambda$, which simulates energy and atom loss. Without damping, the matter waves continue to oscillate in momentum space, while damping leads to the localization in region B (see Supplemental Material, Fig. S4 [36]). We determine the $1 / e$-decay time of $R$ during the second stage with an exponential fit and find good agreement between experiment and simulation for $\Lambda=0.0225$ ([36], Fig. S5). We believe that the energy removal in the experiment is caused by momentum-dependent atom loss. Without periodic driving, we observe an increased loss for atoms in regions with a negative effective mass and dynamical instabilities, which can cause the local collapse of the wave packet and the loss of the atoms (see [36], Fig. S1(c)). However, with periodic driving we 


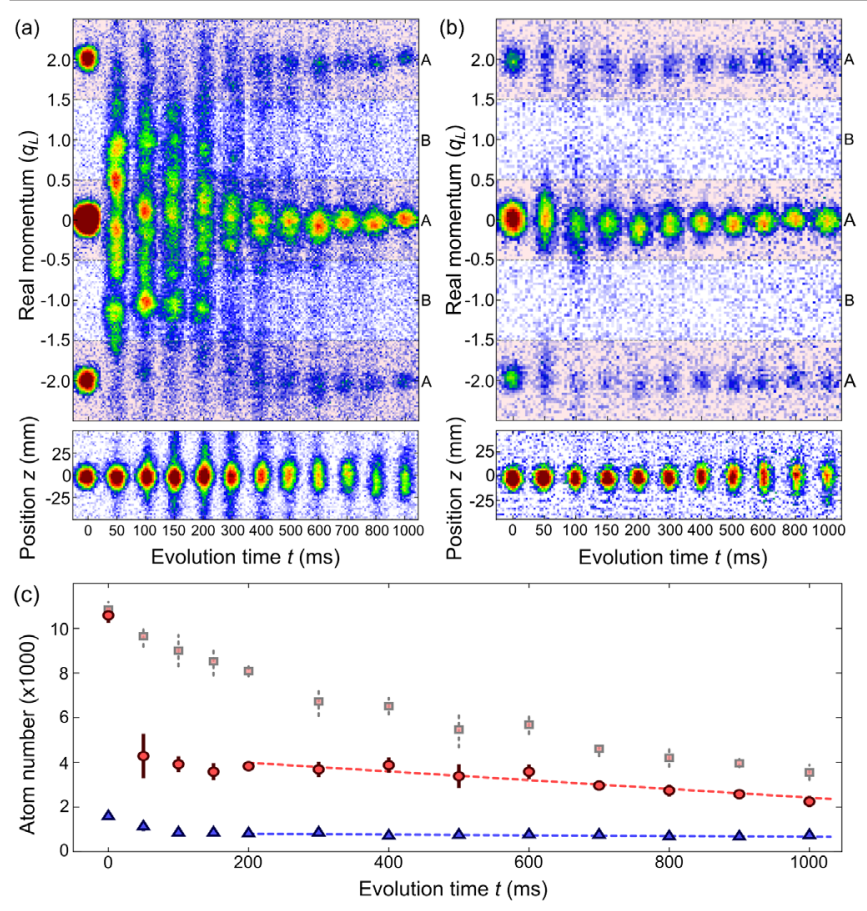

FIG. 3. Matter-wave Floquet solitons. (a) Evolution of wave packet in momentum space (top) and position space (bottom) for a total initial atom number of 11000 and (b) of 1600 [ $\left.V=12.0(5) E_{r}, a_{s}=5 a_{0}, K=4.2\right]$. (c) Atom number in region A for 11000 (red circles) and 1600 (blue triangles) initial atoms. Gray squares provide $N_{\text {tot }}$ for $N_{\text {tot }}(0)=11000$. All measurements are performed without vertical trapping potential.

observe a continuous loss, which is independent of the initial state of the atoms ([36], Fig. S5). This loss might mask other momentum-dependent loss features that cause the damping ([36], Sec. D), and further studies will be necessary.

Removing the trapping potential during the time evolution allows us to study the effects of weak interactions on the wave packet. A negative effective mass causes an effective sign change of the interaction strength $[30,47,50]$. For example, a repulsively interacting wave packet with $q_{0}$ in region $\mathrm{A}$ and $K$ in region II shows the same time evolution as a wave packet with attractive interaction and effective mass $\left|m_{\text {eff }}\right|$. As a result, large interactions lead to dynamical instabilities [33,47,51,52], while weak interactions allow us to observe stable states that show similar properties as bright matter-wave solitons with attractive interaction.

We prepare a wave packet with approximately 11 000 atoms at $a_{s}=5.6 a_{0}$, remove the vertical trapping potential in $1 \mathrm{~ms}$, and study the dispersion of the wave packet for driving strength $K=4.2$ in momentum and position space [Figs. 3(a) and 3(b)]. The wave packet is initially unstable and spreads over the $\mathrm{BZ}$ within the first $200 \mathrm{~ms}$ while shedding atoms along the lattice direction. After $400 \mathrm{~ms}$, a localized wave packet forms at $q_{0}=0$ that is stable for the remaining observation time. The initial
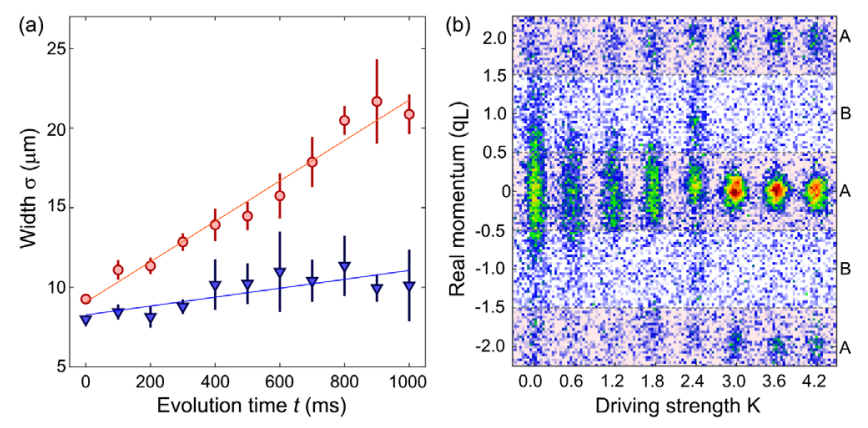

FIG. 4. Matter-wave dispersion. (a) Width of the wave packet in position space in Figs. 3(a) and 3(b) for $N_{\text {tot }}(0)=11000$ (red circles) and 1600 (blue triangles) $\left[V=12.0(5) E_{r}, a_{s}=5.6 a_{0}\right.$, $K=4.2]$. Lines are linear fits with gradients of 12.7(6) (red line) and $2.8(7) \mu \mathrm{m} / \mathrm{s}$ (blue line). (b) Momentum distribution after $500 \mathrm{~ms}$ of driving with for variable driving strength, $N_{\text {tot }}(0)=$ $2000\left[V=12.0(5) E_{r}, a_{s}=3 a_{0}\right]$.

spread is reduced when we lower the atom number to 1600 [Figs. 3(c) and 3(d)], creating a wave packet that is stable both in momentum and position space for the observation time of $1 \mathrm{~s}$.

We interpret those Floquet solitons as matter-wave gap solitons that quickly cycle through the BZ. Gap solitons are bright, nondispersive wave packets with a total energy within the band gap [22,53,54]. They have been experimentally demonstrated for ultracold atoms in nondriven systems close to the edge of the band in region $\mathrm{B}$ where the wave packet has a negative effective mass [23]. For periodic driving, the wave packets evolve due to the time-averaged band energy and the resulting effective mass. Controlling $m_{\text {eff }}$ through time averaging provides another degree of experimental control, and it allows us to create Floquet solitons in other momentum states, e.g., at $q_{0}=0$ for a driving strength in region II.

The density profile $n(z)$ of matter-wave Floquet solitons is given by $n(z)=n_{0} \operatorname{sech}^{2}(z / \sigma)$, where $n_{0}$ is the peak $1 \mathrm{D}$ density and $\sigma$ is the renormalized width of the soliton that depends on the $J_{\text {eff }}$ and on the interaction strength [28]. Using $n(z)$ as a fit function, we demonstrate that the wave packet with 1600 atoms is almost dispersionless with a width of $\sigma=10(3) \mu \mathrm{m}$ at $t=1 \mathrm{~s}$ and a dispersion of 2.8(7) $\mu \mathrm{m} / \mathrm{s}$ [Fig. 4(a)]. The observed solitons are larger than expected ([36], Sec. E), which might be due to our limited imaging resolution, a small thermal background, or residual excitations of the soliton [55].

Finally, we demonstrate the dependence of the wave packet stability on the driving strength $K$ by measuring the spread of the wave packet in momentum space after a driving duration of $500 \mathrm{~ms}$ [Fig. 4(b)]. The wave packet disperses in region I $(K<2.4)$ and remains stable in region II, which indicates that it is indeed the change of signs of effective mass and effective interactions that provide stability.

In summary, we study the dynamics of wave packets with negative effective mass and observe 
three characteristic patterns in the time evolution. First, for a strong suppression of tunneling with $J_{\text {eff }} \approx 0$, the momentum profile shows a multitude of transient patterns, which result from the phase evolution of decoupled wave packets. Second, for sufficiently strong tunneling, the wave packets accumulate at the edge of the $\mathrm{BZ}$ on a timescale that is set by the trapping potential along the lattice direction. We explain this effect by an inversion of the trapping potential due to the negative effective mass and by energy removal due to atom loss. Third, removing the trapping potential allows us to create Floquet solitons that are localized in position and momentum space. Their properties arise from time averaging over the fast periodic micromotion in the first BZ, resulting in an increased stability and experimental control with new opportunities for metrology and matter-wave quantum optics [56]. We expect that other types of lattice solitons [57], e.g., such as discrete breathers, have similar Floquet counterparts in periodically driven systems.

The data used in this publication are openly available at the University of Strathclyde KnowledgeBase [58].

We acknowledge support by the EPSRC through a New Investigator Grant (No. EP/T027789/1), the Programme Grant DesOEQ (No. EP/P009565/1), the Quantum Technology Hub in Quantum Computing and Simulation (EP/T001062/1), and the Programme Grant QSUM (No. EP/P01058X/1).

[1] M. Lewenstein, A. Sanpera, V. Ahufinger, B. Damski, A. Sen(De), and U. Sen, Ultracold atomic gases in optical lattices: Mimicking condensed matter physics and beyond, Adv. Phys. 56, 243 (2007).

[2] C. Gross and I. Bloch, Quantum simulations with ultracold atoms in optical lattices, Science 357, 995 (2017).

[3] A. Eckardt, Colloquium: Atomic quantum gases in periodically driven optical lattices, Rev. Mod. Phys. 89, 011004 (2017).

[4] A. Zenesini, H. Lignier, D. Ciampini, O. Morsch, and E. Arimondo, Coherent Control of Dressed Matter Waves, Phys. Rev. Lett. 102, 100403 (2009).

[5] J. Struck, C. Ölschläger, R. Le Targat, P. Soltan-Panahi, A. Eckardt, M. Lewenstein, P. Windpassinger, and K. Sengstock, Quantum simulation of frustrated classical magnetism in triangular optical lattices, Science 333, 996 (2011).

[6] M. Aidelsburger, M. Atala, S. Nascimbène, S. Trotzky, Y.-A. Chen, and I. Bloch, Experimental Realization of Strong Effective Magnetic Fields in an Optical Lattice, Phys. Rev. Lett. 107, 255301 (2011).

[7] J. Struck, C. Ölschläger, M. Weinberg, P. Hauke, J. Simonet, A. Eckardt, M. Lewenstein, K. Sengstock, and P. Windpassinger, Tunable Gauge Potential for Neutral and Spinless Particles in Driven Optical Lattices, Phys. Rev. Lett. 108, 225304 (2012).
[8] C. V. Parker, L. C. Ha, and C. Chin, Direct observation of effective ferromagnetic domains of cold atoms in a shaken optical lattice, Nat. Phys. 9, 769 (2013).

[9] N. Goldman, G. Juzelinas, P. Öhberg, and I. B. Spielman, Light-induced gauge fields for ultracold atoms, Rep. Prog. Phys. 77, 126401 (2014).

[10] G. Jotzu, M. Messer, R. Desbuquois, M. Lebrat, T. Uehlinger, D. Greif, and T. Esslinger, Experimental realization of the topological Haldane model with ultracold fermions, Nature (London) 515, 237 (2014).

[11] N. Fläschner, B. S. Rem, M. Tarnowski, D. Vogel, D.-S. Luhmann, K. Sengstock, and C. Weitenberg, Experimental reconstruction of the Berry curvature in a Floquet Bloch band, Science 352, 1091 (2016).

[12] K. Wintersperger, C. Braun, F. N. Ünal, A. Eckardt, M. D. Liberto, N. Goldman, I. Bloch, and M. Aidelsburger, Realization of an anomalous Floquet topological system with ultracold atoms, Nat. Phys. 16, 1058 (2020).

[13] M. Holthaus, Floquet engineering with quasienergy bands of periodically driven optical lattices, J. Phys. B 49, 013001 (2016).

[14] D. H. Dunlap and V. M. Kenkre, Dynamic localization of a charged particle moving under the influence of an electric field, Phys. Rev. B 34, 3625 (1986).

[15] M. Holthaus, Collapse of Minibands in Far-Infrared Irradiated Superlattices, Phys. Rev. Lett. 69, 351 (1992).

[16] H. Lignier, C. Sias, D. Ciampini, Y. Singh, A. Zenesini, O. Morsch, and E. Arimondo, Dynamical Control of MatterWave Tunneling in Periodic Potentials, Phys. Rev. Lett. 99, 220403 (2007).

[17] A. Eckardt, M. Holthaus, H. Lignier, A. Zenesini, D. Ciampini, O. Morsch, and E. Arimondo, Exploring dynamic localization with a Bose-Einstein condensate, Phys. Rev. A 79, 013611 (2009).

[18] C. E. Creffield, F. Sols, D. Ciampini, O. Morsch, and E. Arimondo, Expansion of matter waves in static and driven periodic potentials, Phys. Rev. A 82, 035601 (2010).

[19] E. Arimondo, D. Ciampini, A. Eckardt, M. Holthaus, and O. Morsch, Kilohertz-driven Bose-Einstein condensates in optical lattices, Adv. At. Mol. Opt. Phys. 61, 515 (2012).

[20] C. Heinisch and M. Holthaus, Adiabatic preparation of Floquet condensates, J. Mod. Opt. 63, 1768 (2016).

[21] O. R. Diermann and M. Holthaus, Floquet-state cooling, Sci. Rep. 9, 17614 (2019).

[22] K. M. Hilligsøe, M. K. Oberthaler, and K.-P. Marzlin, Stability of gap solitons in a Bose-Einstein condensate, Phys. Rev. A 66, 063605 (2002).

[23] B. Eiermann, T. Anker, M. Albiez, M. Taglieber, P. Treutlein, K.-P. Marzlin, and M. K. Oberthaler, Bright Bose-Einstein Gap Solitons of Atoms with Repulsive Interaction, Phys. Rev. Lett. 92, 230401 (2004).

[24] S. Mukherjee and M. C. Rechtsman, Observation of Floquet solitons in a topological bandgap, Science 368, 856 (2020).

[25] V. V. Konotop and M. Salerno, Modulational instability in Bose-Einstein condensates in optical lattices, Phys. Rev. A 65, 021602(R) (2002).

[26] B. J. Dąbrowska, E. A. Ostrovskaya, and Y.S. Kivshar, Instability-induced localization of matter waves in moving optical lattices, Phys. Rev. A 73, 033603 (2006). 
[27] A. R. Kolovsky, Bright solitons and self-trapping with a Bose-Einstein condensate of atoms in driven tilted optical lattices, Phys. Rev. A 82, 011601(R) (2010).

[28] E. Michon, C. Cabrera-Gutiérrez, A. Fortun, M. Berger, M. Arnal, V. Brunaud, J. Billy, C. Petitjean, P. Schlagheck, and D. Guéry-Odelin, Phase transition kinetics for a Bose Einstein condensate in a periodically driven band system, New J. Phys. 20, 053035 (2018).

[29] S. Arlinghaus and M. Holthaus, Generalized acceleration theorem for spatiotemporal Bloch waves, Phys. Rev. B 84, 054301 (2011).

[30] H. Pu, L. O. Baksmaty, W. Zhang, N. P. Bigelow, and P. Meystre, Effective-mass analysis of Bose-Einstein condensates in optical lattices: Stabilization and levitation, Phys. Rev. A 67, 043605 (2003).

[31] M. Weinberg, C. Ölschläger, C. Sträter, S. Prelle, A. Eckardt, K. Sengstock, and J. Simonet, Multiphoton interband excitations of quantum gases in driven optical lattices, Phys. Rev. A 92, 043621 (2015).

[32] M. Reitter, J. Näger, K. Wintersperger, C. Sträter, I. Bloch, A. Eckardt, and U. Schneider, Interaction Dependent Heating and Atom Loss in a Periodically Driven Optical Lattice, Phys. Rev. Lett. 119, 200402 (2017).

[33] S. Lellouch, M. Bukov, E. Demler, and N. Goldman, Parametric Instability Rates in Periodically Driven Band Systems, Phys. Rev. X 7, 021015 (2017).

[34] K. Singh, C. J. Fujiwara, Z. A. Geiger, E. Q. Simmons, M. Lipatov, A. Cao, P. Dotti, S. V. Rajagopal, R. Senaratne, T. Shimasaki, M. Heyl, A. Eckardt, and D. M. Weld, Quantifying and Controlling Prethermal Nonergodicity in Interacting Floquet Matter, Phys. Rev. X 9, 041021 (2019).

[35] K. Wintersperger, M. Bukov, J. Näger, S. Lellouch, E. Demler, U. Schneider, I. Bloch, N. Goldman, and M. Aidelsburger, Parametric Instabilities of Interacting Bosons in Periodically-Driven 1D Optical Lattices, Phys. Rev. X 10, 011030 (2020).

[36] See Supplemental Material at http://link.aps.org/ supplemental/10.1103/PhysRevLett.127.243603 for a detailed description of experimental parameters, which includes Refs. [37-41].

[37] A. D. Carli, C. D. Colquhoun, S. Kuhr, and E. Haller, Interferometric measurement of micro- $g$ acceleration with levitated atoms, New J. Phys. 21, 053028 (2019).

[38] A. I. Yakimenko, K. O. Isaieva, S. I. Vilchinskii, and M. Weyrauch, Stability of persistent currents in spinor BoseEinstein condensates, Phys. Rev. A 88, 051602(R) (2013).

[39] A. Di Carli, G. Henderson, S. Flannigan, C. D. Colquhoun, M. Mitchell, G.-L. Oppo, A. J. Daley, S. Kuhr, and E. Haller, Collisionally Inhomogeneous Bose-Einstein Condensates with a Linear Interaction Gradient, Phys. Rev. Lett. 125, 183602 (2020).

[40] E. Haller, R. Hart, M. J. Mark, J. G. Danzl, L. Reichsöllner, and H.-C. Nägerl, Inducing Transport in a Dissipation-Free Lattice with Super Bloch Oscillations, Phys. Rev. Lett. 104, 200403 (2010).

[41] A. Smerzi and A. Trombettoni, Nonlinear tight-binding approximation for Bose-Einstein condensates in a lattice, Phys. Rev. A 68, 023613 (2003).
[42] O. Morsch and M. K. Oberthaler, Dynamics of BoseEinstein condensates in optical lattices, Rev. Mod. Phys. 78, 179 (2006).

[43] M. A. Khamehchi, K. Hossain, M. E. Mossman, Y. Zhang, T. Busch, Michael McNeil Forbes, and P. Engels, NegativeMass Hydrodynamics in a Spin-Orbit-coupled Bose-Einstein Condensate, Phys. Rev. Lett. 118, 155301 (2017).

[44] A. E. Kaplan, I. Marzoli, W. E. Lamb, and W. P. Schleich, Multimode interference: Highly regular pattern formation in quantum wave-packet evolution, Phys. Rev. A 61, 032101 (2000).

[45] M. Gustavsson, E. Haller, M. J. Mark, J. G. Danzl, R. Hart, A. J. Daley, and H.-C. Nägerl, Interference of interacting matter waves, New J. Phys. 12, 065029 (2010).

[46] M. J. Mark, E. Haller, J. G. Danzl, K. Lauber, M. Gustavsson, and H.-C. Nägerl, Demonstration of the temporal matterwave Talbot effect for trapped matter waves, New J. Phys. 13, 085008 (2011).

[47] C. E. Creffield, Instability and control of a periodically driven Bose-Einstein condensate, Phys. Rev. A 79, 063612 (2009).

[48] S. Choi, S. A. Morgan, and K. Burnett, Phenomenological damping in trapped atomic Bose-Einstein condensates, Phys. Rev. A 57, 4057 (1998).

[49] A. Rançon and K. Levin, Equilibrating dynamics in quenched Bose gases: Characterizing multiple time regimes, Phys. Rev. A 90, 021602(R) (2014).

[50] N. Tsuji, T. Oka, P. Werner, and H. Aoki, Dynamical Band Flipping in Fermionic Lattice Systems: An ac-Field-Driven Change of the Interaction from Repulsive to Attractive, Phys. Rev. Lett. 106, 236401 (2011).

[51] B. Wu and Q. Niu, Landau and dynamical instabilities of the superflow of Bose-Einstein condensates in optical lattices, Phys. Rev. A 64, 061603(R) (2001).

[52] L. Fallani, L. De Sarlo, J. E. Lye, M. Modugno, R. Saers, C. Fort, and M. Inguscio, Observation of Dynamical Instability for a Bose-Einstein Condensate in a Moving 1D Optical Lattice, Phys. Rev. Lett. 93, 140406 (2004).

[53] S. K. Adhikari and B. A. Malomed, Gap solitons in a model of a superfluid fermion gas in optical lattices, Physica (Amsterdam) 238D, 1402 (2009).

[54] P. Kizin, D. Zezyulin, and G. Alfimov, Oscillatory instabilities of gap solitons in a repulsive Bose-Einstein condensate, Physica (Amsterdam) 337D, 58 (2016).

[55] A. Di Carli, C. D. Colquhoun, G. Henderson, S. Flannigan, G.-L. Oppo, A. J. Daley, S. Kuhr, and E. Haller, Excitation Modes of Bright Matter-Wave Solitons, Phys. Rev. Lett. 123, 123602 (2019).

[56] H. Sakaguchi and B. A. Malomed, Interactions of solitons with positive and negative masses: Shuttle motion and coacceleration, Phys. Rev. E 99, 022216 (2019).

[57] Y. V. Kartashov, B. A. Malomed, and L. Torner, Solitons in nonlinear lattices, Rev. Mod. Phys. 83, 247 (2011).

[58] M. Mitchell, A. Di Carli, G. Sinuco-León, A. La Rooij, S. Kuhr, and E. Haller, Data for "Floquet Solitons and Dynamics of Periodically Driven Matter Waves with Negative Effective Mass", University of Strathclyde KnowledgeBase, https://doi.org/10.15129/6d483da1-0678403c-be63-011f9ecd77f0. 